\title{
One Approach to Idioms and Their Peculiarities in the English Language
}

\author{
Nino Kemertelidze, Professor \\ Georgian Technical University, Tbilisi, Georgia \\ Meri Giorgadze, PhD \\ Ivane Javakhishvili Tbilisi State University, Tbilisi, Georgia
}

Doi:10.19044/esj.2020.v16n11p13～ＵRL:http://dx.doi.org/10.19044/esj.2020.v16n11p13

\begin{abstract}
The presented article is dedicated to a very interesting phenomenon existing in linguistics and literary theory - idiom. It aims to study some peculiarities of idioms and identify their different classes which were revealed while processing the material under investigation; to categorize and regulate the idioms united in these classes according to strength, motivation and informativity. The study was grounded on two types of classification of idioms (classification according to their usage and semantic classification), comparative analysis of these two classifications and the conducted experiment. The data the research was based on included 70 randomly chosen idioms. Another objective of the study is to reveal how this or that idiom is perceived by people hence, generally, it is difficult to comprehend the exact meaning of majority of idioms even for native speakers. Most of them find it hard to resolve the real sense of an idiom as in some cases its literal meaning is different from its figurative one. The conducted analysis is dictated and conditioned namely by this fact. It is a well-known fact that idiom is a set word-combination the meaning of which is not determined by the meaning of the words included in its composition. The findings of the presented study reveal that a pure idiom should necessarily meet the following formula: strong idiom $=$ non-informative + non-motivated. The analysis also showed that out of 70 randomly chosen idioms, about half of them (34 idioms) follow the mentioned formula, though, there are different cases of deviation from this formula.
\end{abstract}

Keywords: Idiom, informativity, strength, motivation

\section{Introduction}

Idiom is a Greek word and means odd, peculiar expression. It is a set word-combination the meaning of which is not determined by the meaning of 
the words included in its composition. Idiom should necessarily consist of at least two words.

Idiom has always been attracting researchers' attention. A lot of things have been written and are still being written about this phenomenon in linguistics and literary theory. The presented article discusses idioms in quite a different aspect. It aims to discuss some peculiarities of idioms and identify their different classes which were revealed while processing the material under investigation. The data the research was based on included 70 randomly chosen idioms under two domains (food and parts of body idioms).

The research deals with the classification of idioms according to their usage as well as with their semantic classification based on the degree of strength, informativity and motivation revealed in each idiom under investigation. Based on the method of comparative analysis, the article also focuses on correlation between these two types of classification.

Semantic classification is based on the conducted experiment. Subjects of the experiment were 50 English-speaking highly qualified professionals. Each of them was given 70 randomly selected idioms. Their task was to express the emotion this or that idiom evoked in them and consequently, mark each idiom as "strong", "not strong enough" or " weak". In addition to determining the main component of the semantic classification, they also had to estimate the degree of informativity and motivation of each idiom. About 85 percent of the subjects considered 37 idioms as strong, 18 - as not strong enough and 15 - as weak.

\section{Classification of idioms according to their usage:}

The analysis of idioms according to their usage revealed three classes:

Class I: The first class united the idioms the constituent parts of which can also be used as free word-combinations, though, such a collocation does not exist unless being an idiom. For instance:

1) The phrase "hot potato" as an idiomatic expression refers to a problem, situation or issue that is difficult to deal with and causes a lot of disagreements: "The bombing of Syria is a political hot potato." The mentioned phrase can also be used as a free word-combination that implies potato that is hot: "I can't eat potatoes that are too hot".

2) The idiom "lay an egg" means to fail, especially in a public performance; to make a humiliating error or do something unsuccessful: "Yesterday Bob laid an egg when he forgot his lines." The literal meaning of the same phrase is quite different: "Our hen has just laid an egg”. In this case, we deal with a free word-combination.

3) The expression "a piece of cake" describes something that is easy to achieve: "I'm sure the test next week will be a piece of cake for me. I've been studying for weeks". The mentioned expression can also be 
used a free word-combination as it is given in the following example: "The boy snatched a piece of cake and ate it greedily".

Class II: Idioms belonging to the second class are polysemantic bound wordcombinations. It means that a word-combination has an idiomatic meaning, and on the other hand, is merely a set phrase.

1) The expression "head over heels" is a set phrase that does not coincide with the corresponding free word-combination. Its idiomatic meaning is: to be completely in love. "I have been head over heels about my girlfriend since the day I met her." On the other hand, the same combination of words has another meaning: upside down: "When I entered the room, I saw that everything was head over heels". In latter case the word-combination is bound though not an idiom.

2) The set phrase "play by ear" has the following meanings: a) To play a piece of music without referencing sheet music or a recording: "I found I could just sit down and play by ear"; b) To make decisions about what action to take in an adaptive, flexible way, based on the circumstances: "What time shall we go shopping?" "Let's see how the weather looks and play it by ear."

Class III: The third class comprises idioms that are monosemantic bound word-combination. It means that a word-combination of this class is used only as an idiom.

1) The expression "pie in the sky" implies something that you hope will happen but is very unlikely to happen: "To complete the task in one day is a pie in the sky." The meaning of the mentioned wordcombination in any other sense is absurd, illogical.

2) The phrase "coach potato" is an idiom that means an idle person who does not do anything but watches TV: "Bob, you are a typical couch potato. You'd better be more active." The expression does not have any other logical meaning.

Thus, the analysis showed that out of 70 randomly chosen idioms 26 of them are united in the $1^{\text {st }}$ class - idioms that can be used as a free wordcombination, though such a collocation does not exist unless being an idiom; 38 idioms are discussed under the $2^{\text {nd }}$ class - a word-combination has an idiomatic meaning, and on the other hand, is merely a set phrase; 6 idioms are included in the $3^{\text {rd }}$ class of the above-mentioned category.

\section{Semantic classification:}

The idioms under investigation were studied in the context of informativity, motivation and strength. Though motivation and informativity 
of idioms have been studied by different linguists (Dobrovol'skij D., Piirainen E., 2010), (Kovacs G., 2016), (Panou D., 2013), these researches discuss motivation and informativity of idioms in absolutely different aspect. The presented study analyses idioms in the context of informativity, motivation and strength not discretely, but collectively. Such approach is the first attempt in the study of idioms and can be considered as the novelty presented in the research.

\section{Informativity:}

Under informativity is implied the extent to which the content of a word-combination is already known or expected, compared with the unknown or unexpected. In determining informativity, pragmatic aspect plays an essential role, i.e. relation of the content of the text to the knowledge available to the reader/ listener/ addressee on this issue.

While studying the idioms under investigation we came across the idioms which, based on their constituent components, appeared to be noninformative, partially informative and informative.

Let us discuss each case separately.

Non-informative - we consider that an idiom is non-informative if neither of its components provides us with any kind of information or gives us even a hint concerning the meaning of the idiomatic expression. For instance:

1) The idiom "an arm and a leg" is used to describe anything that is considered to be very expensive or excessively costly. The explanation of our supposition that this idiomatic expression is noninformative lies in the fact that none of its constituents gives us any clue regarding its meaning as an idiom. Thus, the meaning of the idiom "an arm and a leg" is not at all connected to body parts - an arm and a leg.

2) The idiom "the apple of one's eye" is defined in Collins English Dictionary (2011) as a person or a thing that is very precious and loved. Though the idiomatic expression originally had anatomical reference and was used to denote the central aperture of the eye, it is still impossible to predict its meaning from the meanings of the constituent words of the idiom.

3) It is also unpredictable to guess the meaning of the following idiom "foot in mouth" as the literal meaning of its constituents is absolutely different from the idiomatic meaning that sounds as follows: to say or do something accidentally offensive or embarrassing. The example "She put her foot in her mouth" (she said something offensive) has the same grammatical structure as a neutral sentence such as, "She put her apple in her mouth" (the apple that was hers). The difference is that in 
the second case, everybody understands the sentences quite easily but in the first case, nobody guesses its meaning unless one knows it as an idiom.

Partially informative - we suggest that an idiom can be partially informative if at least one of its components gives us the clue about the meaning of an idiom. For instance:

1) The idiom " $a$ sweet tooth" refers to a person who has a strong craving to eat sweets, in other words, someone who is a big lover of sweets. Decoding the meaning of the idiom is not very difficult as the semantic field of its constituent elements is related to the actual meaning of the idiom. The meaning of the word "sweet" (having a taste similar to that of sugar) can give a reader a kind of hint. Based on this fact, we consider the mentioned idiom partially informative.

2) The meaning of the idiom "play into one's hands" is to do something that gives others an advantage over you or helps an opponent to defeat you. We consider the idiom partially informative due to the meaning of the words "play" and "hand". Based on the origin of the above mentioned idiom, the expression is connected to the game of cards. As it is known, a part of the game's strategy is in your hands and defines the follow-up strategies on your opponent's side. "Hands" as body parts are seen as the instrument of control.

3) The idiom "itchy feet" refers to the desire and willingness one has to move from one place to another or travel a lot; also to be restless and change or start something new. What makes us consider the idiom partially informative is the meaning of the second constituent word of the idiom. The word "feet" is typically associated with the movement from one place to another. Thus, the nominative meaning of the word "feet" gives us a certain hint.

Informative - we consider that an idiom is informative if all its constituent elements give the definite idea about the idiomatic expression. For instance:

1) The idiomatic expression "heart of gold" implies a person who is very kind, generous and caring. The idiom is supposed to be informative as each constituent element gives a clue and makes it easy for the addressee to guess its connotation. According to its origin, it comes from the idea of gold being a precious metal that is highly valued. In this sense, a person with a heart of gold refers to someone who has a heart which is valued for its goodness and kindness.

2) The idiom "use one's noodle" conveys the following meaning: use one's brain, intelligence and an intellectual ability. We count it informative as long as its idiomatic meaning coincides with the literal 
meaning of the idiom. One of the meanings of the word "noodle" is brain, head. It is used as slang and every English-speaking person is aware of it. Thus, the meaning of the expression as an idiom can simply be predicted.

3) The meaning of the idiomatic expression "burn one's fingers" is to suffer unpleasant and ruinous consequences; especially, it is used in a financial context. Certainly, it is not pleasant to burn one's fingers. We have been warned since early childhood not to touch anything dangerous or hot to avoid burning our fingers. So, indication and a hint (that it is associated with something unpleasant) is clearly given in the idiom. That is why we discuss it under the category of informative idioms.

Thus, based on the above discussed examples, in respect of noninformative idioms, neither of the components of the idiomatic expression has a primary informative function considering the meaning of the idiomatic expression itself.

In the case of partially informative idioms, idiomatic construction is partially clear for the addressee regarding the semantic meanings of its constituents.

As for informativity, it is a fairly common case when the content of a word-combination is already known or expected, compared with the unknown or unexpected. In other words, the word combination in this case is mostly semantically transparent.

\section{Motivation:}

In the given research, under motivation is meant the logical relation between the constituent parts of an idiom.

We classified all idioms under investigation into three categories: nonmotivated, partially motivated and motivated.

Let us discuss each case separately:

Non-motivated - we consider that an idiom is non-motivated if there is no logical relation between its constituent parts in the sense of an idiom. For instance:

1) "Full of beans" as an idiom means to have much energy and enthusiasm; to be in high spirits. We count the idiom non-motivated as its idiomatic meaning is completely distant from the meanings of its constituent words. The constituent components are used figuratively and give no hint at all to guess its idiomatic connotation.

2) The idiom "chalk and cheese" stands for something that is absolutely different, opposite. The mentioned idiom is a synonym of the idiom 
"apples and oranges". Both of them are considered to be nonmotivated as their word for word translation is radically different from the idiomatic meaning and there is no logical connection between them. Thus, none of the idioms gives a reader or a listener any clue to postulate their idiomatic meaning.

Partially motivated - we consider that an idiom is partially motivated if the logical relation between its components is not vividly perceived. For instance:

1) The idiom "big cheese" refers to someone who is influential and has an important and powerful position in an organization. Due to the meaning of one of its constituent elements (cheese) the idiom is partially motivated. As a slang, it means someone important or special.

2) The idioms "tough cookie" (a strong, determined person who is not easily intimidated, discouraged, or defeated) and "smart cookie" (a clever and intelligent person) are considered to be partially motivated as the word "cookie", one of the components of the idioms, gives us the clue. As a slang, it refers to someone, a person of a specified kind; a guy.

3) The same can be said about the following idiomatic expressions: "bad egg" (an individual who turns out to be rotten), "bad/rotten apple" (a person whose own words or actions negatively impact an entire group of people). In these cases, we have twofold hints. The adjectives "bad" and "rotten" have negative connotations and the words "egg" and "apple" as slangs mean a person of a specified kind.

Thus, one who is aware of the aforementioned meanings will find some kind of connection between the constituent elements of the idioms though it is definitely not vivid.

Motivated - we consider that an idiom is motivated if the logical relation between its constituent parts is more or less apparent. For instance:

1) The idiom "flesh and blood" stands for someone who is your family member or a close relative. Based on the semantically motivated constituent elements, we regard this idiomatic expression motivated what means that the meaning of the idiom can be inferred from its components. Flesh and blood are inseparable in a living being: flesh is the soft substance that consists of muscle and fat and blood - liquid that circulates in the arteries and veins of a living creature.

2) The motivation of the idiom "come to/into (one's) head" (to reach a point in a situation where action needs to be taken; a culmination of events leading to a conclusion) is caused by the fact that the meaning 
of the idiomatic expression and the meaning of its component parts are mostly the same in its literal sense. The meaning is obvious and, consequently, we regard it as motivated.

3) We suppose that the idiom "zip one's lip (mouth)" (to close one's mouth and be quiet; not to reveal a secret) is motivated because there is an obvious link between the meaning of the whole unit and the meanings of its components. The word "zip" directly gives the hint that means to close, to fasten.

Consequently, in case of non-motivated idioms, neither of the components of the idiomatic expression has initial, provoking loading in the sense of an idiom itself.

As for the partially motivated idioms, idiomatic construction is partially penetrable to its addressee regarding the logical relation of its constituents.

Considering the above discussed examples, idioms are counted to be motivated when there is a logical connection between its constituent elements that enables a reader or a listener to guess the inferred meaning of the idiom.

\section{Strength:}

Under the strength of an idiom we imply the emotion that it provokes in a person. This category is definitely subjective as can vary from person to person. Its perception depends on a person's emotional cognition, his/her world outlook and world image.

Based on the results of the carried out experiment, we divided the idioms under investigation into three classes: strong, not strong enough and weak.

Let us discuss each case separately:

Strong - we consider that an idiom is strong if it arises strong emotions in most people. For instance:

The idiom "head in the clouds" refers to a person who is daydreaming or absent-minded, who is unaware what is going on. All the subjects participating in the experiment considered this idiom as strong. Having one's head in the clouds is definitely impossible what provoke certain emotions in the subject.

The same can be said about the idiomatic expression "head over heels" which is used when a person is completely in love. It seems that namely the semantic meaning of the whole phrase caused great emotions in the members of the target group.

Not strong enough - we consider that an idiom is not strong enough if the emotion arisen by it is not as strong as in the case of strong idioms. For instance: 
The idiomatic expression "brain(s) trust" describes a group of close advisers to a political candidate or incumbent. The great majority of the subjects of the experiment considered the idiom to be not strong enough as the direct meaning of the expression more or less corresponds to its idiomatic meaning and causes the emotion which is not that much strong. According to the most subjects, the idiom "neck and neck" (extremely near one another) also belongs to the class of idioms "not strong enough". Though this idiom originally was a sport idiom, it is still used in ordinary speech.

Weak - we consider that an idiom is weak if it does not arise emotions in most people. For instance:

As most of the subjects noted, the idiom "wag (one's) chin" (to talk or jabber; to chatter aimlessly) did not arise any emotions in them. Thus, we can include it in the class of "waek" idioms.

Hence, according to the carried out experiment and conducted research, strong idioms are more unexpected and consequently, arise strong emotions. The level of emotion decreases in the idioms united under the class "not strong enough" and those that are united under the class "weak" do not arise any emotions.

Thus, out of randomly chosen 70 idioms belonging to the semantic fields of food and parts of body, 37 idioms appeared to be strong, 18 of them - not strong enough and 15 - weak.

\section{Detailed analysis of idioms in terms of their strength:}

It is supposed that strong idioms should be non-motivated and noninformative, but the analysis showed that this is not always the case. It appeared that out of 37 idioms belonging to the class of strong idioms, 34 of them follow the aforementioned formula (strong idiom = non-motivated + non-informative), though some exceptions were identified. For instance:

a) We came across two cases when idioms united under the group "strong" are non-informative, but partially motivated (one component [noninformative] coincides with the above-mentioned formula, the second component is partially different).

1) The meaning of the idiom "cream of the crop" is being the best of something; the best of the best. As it seems from our research, one component [non-informative] coincides with the formula and the second component is partially different, since it is partially motivated instead of being non-motivated.

2) The idiomatic expression "high tea" implies a meal eaten in the late afternoon or early evening, typically consisting of a cooked dish, bread and butter, and tea. A possible explanation why this type of meal was called "high tea" is the fact that it was eaten at a table. Based on our study, one component [non-informative] coincides with the 
formula and the second component is partially different, since it is partially motivated instead of being non-motivated.

b) While carrying out the research we found one case when a strong idiom appeared to be non-motivated, but informative (one component [nonmotivated] coincides with the formula, the second component [informative] is radically different).

1) An idiom "neck of the woods" refers to a nearby area or neighborhood; the area around where one lives. In the given idiom only one component [non-motivated] coincides with the mentioned formula, while the second component [informative] is radically different.

Figure 1. graphically expresses the aforementioned:

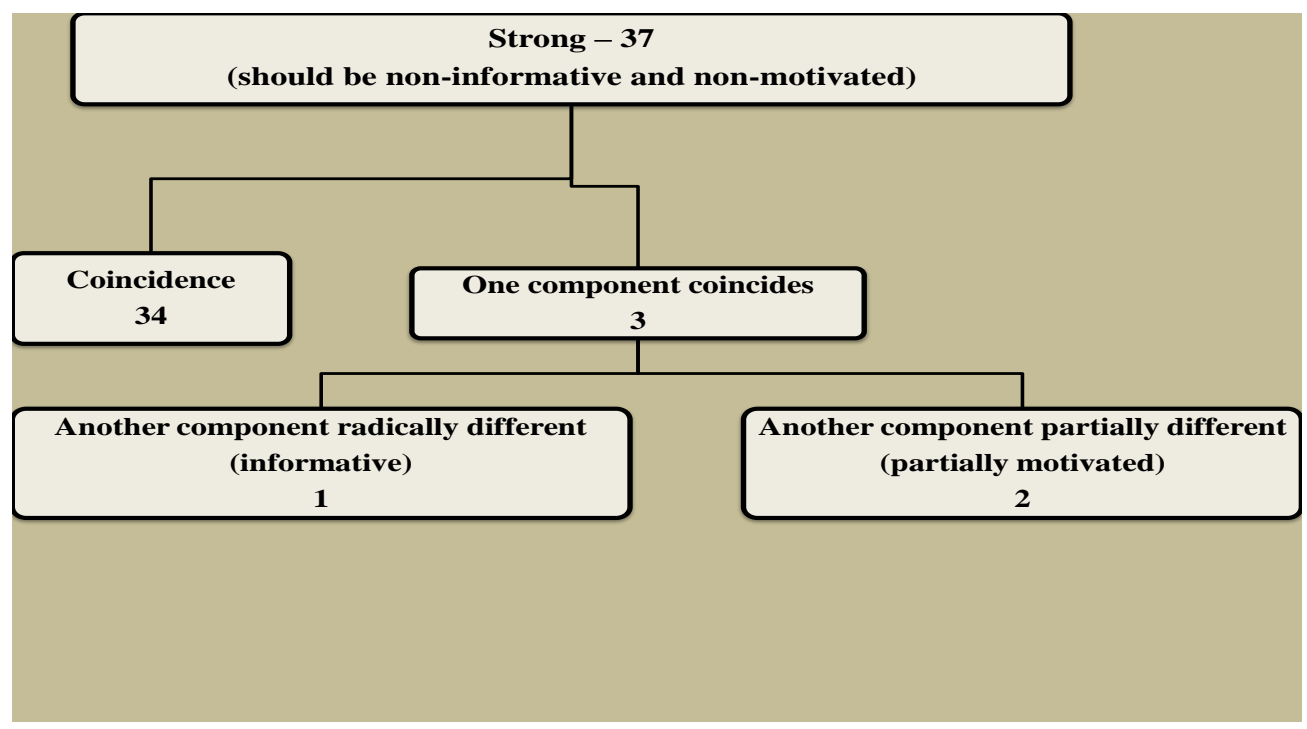

It is supposed that weak idioms should be motivated and informative, but the analysis showed that this is not always so. It appeared that out of 15 idioms belonging to the class of weak idioms, 9 of them follow the aforementioned formula ( weak idiom $=$ motivated + informative $)$, though some exceptions were identified. For instance:

a) During the analysis four cases of informative but non-motivated strong idioms were observed (one component [informative] coincides with the aforementioned formula, but the second component [non-motivated] is radically different).

1) The meaning of the idiom "pull (one's) leg" is to tease or fool someone when trying to convince him/her to believe something which is not true. Considering the formula worked out in the research, in the aforementioned idiom one component [informative] coincides with the formula, but the second component [non-motivated] is radically different. 
2) The "twist one's arm" means to pressure or force one into doing something. Its figurative meaning is the image of hurting someone until they agree to cooperate. Based on the research study, only one component [informative] coincides with the formula, while the second one [non-motivated] is radically different.

b) We also came across two cases when strong idioms are informative, but partially motivated (one component [informative] coincides with the formula, but the second one is partially different, to be exact - partially motivated).

1) The phrase "body and soul" as an idiom means physically and mentally; involving every aspect of a person; completely. It is clear that only one component [informative] coincides with the formula, but the second component [partially motivated] is partially different.

2) The idiom "a bag of bones" indicates a state when one has a frail appearance;

a person or animal that is emaciated to the point that bones can be see $\mathrm{n}$ protruding from the skin. On the report of our experiment, only one component [informative] coincides with the mentioned formula, while the second one [partially motivated] is partially different.

Figure 2. graphically expresses the aforementioned:

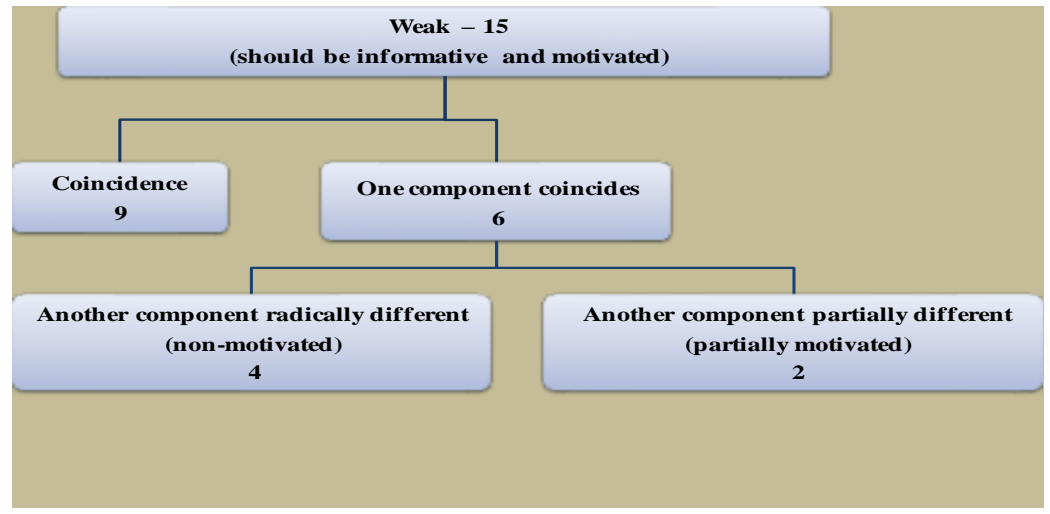

As for the group uniting the idioms which are not strong enough, it was not quite possible to have prior supposition, though, based on the above given formulas, we can suppose that the formula of the idioms included in this group should be partially motivated and partially informative, but the analysis showed that this is not always the case. It appeared that out of 18 idioms belonging to the class of not strong enough idioms, 9 of them follow the aforementioned formula (not strong enough idiom = partially motivated + 
partially informative), though some exceptions were still observed. For instance:

a) We came across two cases which are radically different from the worked out formula (non-informative and non-motivated idioms).

1) The idiom "rule of thumb" infers a principle that is kept to; a guide that is based on practice rather than theory. In the framework of the research, the idiom is radically different from the formula, as it is neither partially motivated nor partially informative. It is noninformative and non-motivated.

b) During the analysis we met seven cases of partially informative, but non-motivated idioms though they are not strong enough (one component [partially informative] coincides with the formula, the second one [nonmotivated] is radically different).

1) The idiom "find (one's) feet" indicates a state when one reaches a level of comfort in a new situation. On the report of the carried out experiment, only one component [partially informative] coincides with the formula, the second one [non-motivated] is radically different.

2) The phrase "bread and butter" as an idiom refers to a job or activity that provides one with the money needed for living; figuratively, it is someone's basic income; a vital component of something. As it is clear, only one component [partially informative] coincides with the formula, the second one [non-motivated] is radically different).

3) We have exactly the same illustration in the case of the idiomatic expression "bread and

4) water" that means the bare essentials for sustenance; figuratively, it is the most minimal meal possible. 
Figure 3. graphically expresses the aforementioned:

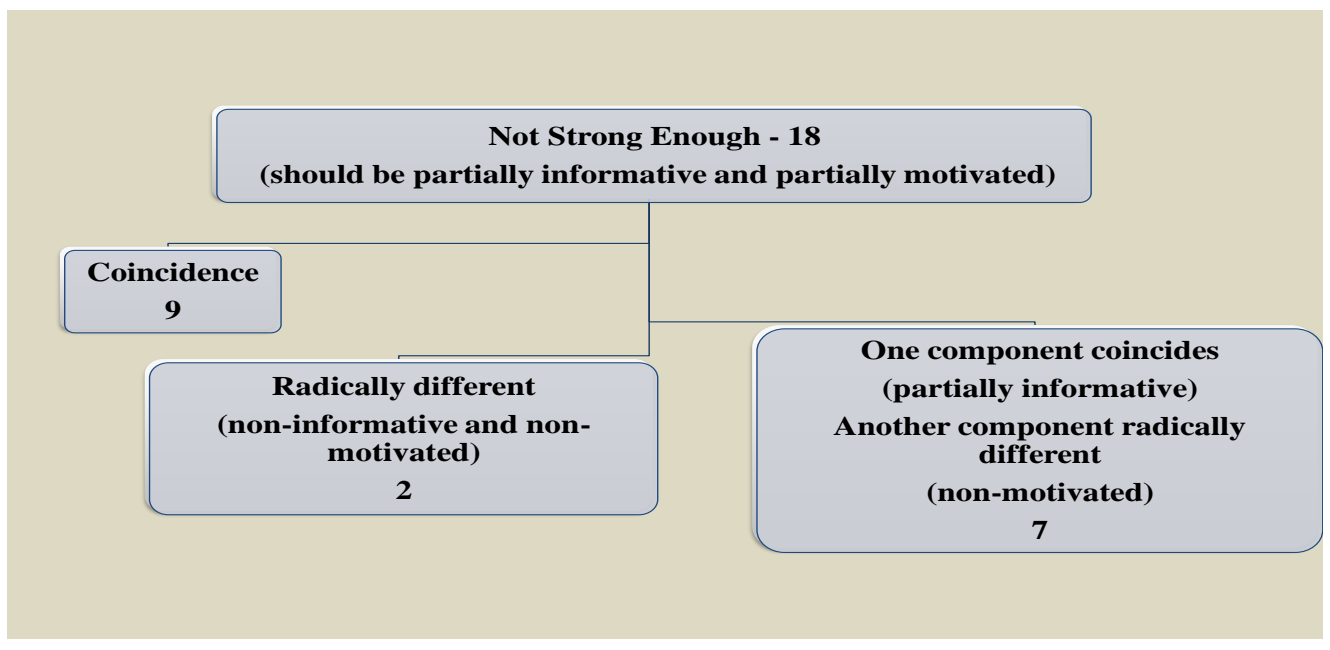

Thus, in this respect, idioms can be classified as a) informative, partially informative, non-informative; b) motivated, partially motivated, nonmotivated; c) strong, not strong enough, weak. After studying the material, we can come to the conclusion that idioms that follow the formula strong idiom $=$ non-motivated + non-informative can be considered as pure idioms . The conducted analysis revealed 34 such cases out of 70 idioms. 
Figure 4. vividly describes the whole classification and the interrelation between the mentioned classes.

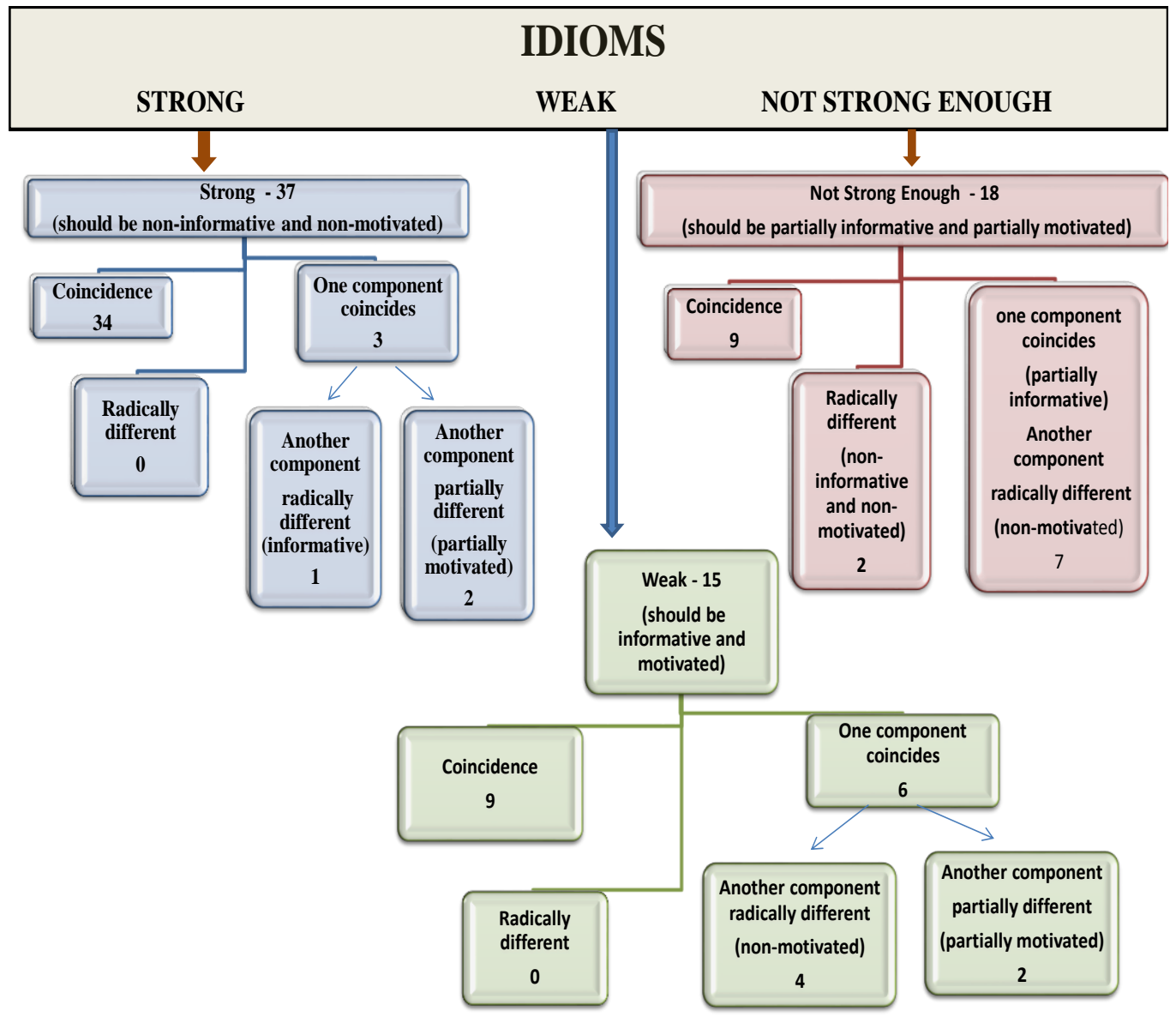

\section{Comparative analysis:}

The method of comparative analysis showed the correlation between the two types of classification discussed above: 1) classification according to the usage of idioms; and 2) semantic classification. The analysis revealed that most of the strong idioms (20 idioms) are united in the $3^{\text {rd }}$ class of the classification according to their usage, i.e. monosemantic idioms. The $1^{\text {st }}$ class, i.e. when an idiomatic word-combination can also be used as a free wordcombination comprises 13 strong idioms and the $2^{\text {nd }}$ class, i.e. polysemantic idioms -4 strong idioms. 
Figure 5. vividly shows the mentioned correlation:

Class I - 26 Idioms

-Strong - 13

-Not strong enough - 6

-Weak - 7
Class II - 6 Idioms

-Strong - 4

-Not strong enough - 2

-Weak - 0
Class III - 38 Idioms

-Strong - 20

- Not strong enough - 10

-Weak - 8

\section{Conclusion}

Thus, the research was based on two types of classification of idioms (classification according to their usage and semantic classification), comparative analysis of these two classifications and the conducted experiment.

The findings of the presented study reveal that a pure idiom should necessarily meet the following formula: strong idiom $=$ non-informative + non-motivated. The analysis also showed that out of 70 randomly chosen idioms, about half of them (34 idioms) follow the mentioned formula, though, there are different cases of deviation from this formula.

\section{References:}

1. Ayto, J. (2010). Oxford Dictionary of Idioms. Oxford University Press. Third edition. Bortfield, H. (2003). Comprehending idioms crosslinguistically. Experimental Psychology, 50(3):217-30.

2. Brewer, M. B., Hewstone, M. (2004). Emotion and Motivation. Publisher: Wiley. Bu, J. (2018). Class-Book of Comparative Idioms: English - French. Sagwan Press.

3. Collins English Dictionary. (2011). Collins; 11th Revised edition.

4. Dąbrowska, A. (2019). A Syntactic Study of Idioms. Cambridge Scholars Publishing.

5. Dobrovol'skij D., Piirainen E. (2010). Idioms: Motivation and Etymology. Yearbook of Phraseology, 1:73-96.

6. Fellbaum, Ch. (2007). Idioms and Collocations: Corpus-based Linguistic and Lexicographic Studies. London: Continuum.

7. Fussell, S. R. (2002). The Verbal Communication of Emotions: Interdisciplinary Perspectives. Publisher: Lawrence Erlbaum Associates.

8. Keizer, E. (2018). Idiomatic Expressions and Anaphoric Reference in Functional Discourse

Grammar. https://ojs.uv.es/index.php/qfilologia/article/view/13527/12689 
9. Kovacs G. (2016). About the Definition, Classification, and Translation Strategies of Idioms. Acta Universitatis Sapientiae, Philologica, 8, 3; 85-101.

10. McClure, S. (2011). Modification in non-combining idioms. Semantics and Pragmatics, 4: 1-7.

11. Panou D. (2013). A Study of Idiom Translation from English in the Greek Press. Thesis submitted for the degree of Doctor of Philosophy in Applied Linguistics and TESOL at the University of Leicester. https://pdfs.semanticscholar.org/dc81/0c621393cd6b0dda26b6241e5 3ee5f30c2f2.pdf

12. Pinnavaia, L. (2018). Food and Drink Idioms in English: "A Little Bit More Sugar and Lots of Spice”. Cambridge Scholars Publishing. 